\title{
DIFFERENTIAL OPERATORS ON THE PROJECTIVE PLANE
}

O.A. Torshina, Nosov Magnitogorsk State Technical University, Magnitogorsk, Russian Federation, olganica@mail.ru.

A formula for the addition of even spherical harmonics was obtained in [1]. This formula became the starting point for the derivation of the first regularized trace for the Laplace - Beltrami operator with potential on the projective plane. Due to this formula, it is not need to find asymptotic formulas for the associated Legendre polynomials by the three parameters, which was an impossible problem for a long time. The obtained results are the basis for the calculation of the perturbation theory corrections and allow the following application for the formulas for regularized traces of elliptic differential operators. In this paper we consider the problem of summation of divergent series class. We propose a method for calculating of the corrections of perturbation theory for a differential operator with potential on the real projective plane. The method is applicable, in particular, to take sum of series with factorial growth of its members.

Keywords: operator Laplace - Bochner; projective plane; Hilbert space; Legendre polynomial; Lipschitz condition.

\section{Addition Theorem}

Consider spherical function of order $n[9]$ :

$$
P_{n}(\cos \alpha)=2 \sum_{l=0}^{n} \frac{(n-l) !}{\delta_{l}(n+l) !} P_{n}^{(l)}\left(\cos \theta^{\prime}\right) P_{n}^{(l)}(\cos \theta) \cos l\left(\varphi-\varphi^{\prime}\right),
$$

where $\alpha$ is an angle between the radius vectors in spherical coordinates $(\theta, \varphi)$ and $\left(\theta^{\prime}, \varphi^{\prime}\right)$;

$$
\cos \alpha=\sin \theta \sin \theta^{\prime} \cos \left(\varphi-\varphi^{\prime}\right)+\cos \theta \cos \theta^{\prime}
$$

$\delta_{l}=2$ at $l=0$ and $\delta_{l}=1$ at $l>0$;

$$
P_{n}^{(l)}(x)=\left(1-x^{2}\right)^{l / 2} \frac{d^{l} P_{n}(x)}{d x^{l}}=\frac{\left(1-x^{2}\right)^{l / 2}}{2^{n} n !} \frac{d^{n+l}}{d x^{n+l}}\left[\left(x^{2}-1\right)^{n}\right],
$$

Legendre polynomial

$$
P_{n}(x)=\frac{1}{2^{n} n !} \frac{d^{n}}{d x^{n}}\left[\left(x^{2}-1\right)^{n}\right], \quad-1<x<1 .
$$

Using the addition theorem for Legendre polynomials [11, 13], we write even spherical functions. We assign the negative superscript to the functions which contain $\cos (l \varphi)$, and the positive superscript to $\sin (l \varphi)$.

$$
\begin{array}{ll}
Y_{n}^{(-l)}(\theta, \varphi)=P_{n}^{(l)}(\cos \theta) \cos (l \varphi), & n \equiv 0(\bmod 2), \\
Y_{n}^{(l)}(\theta, \varphi)=P_{n}^{(l)}(\cos \theta) \sin (l \varphi), & n \equiv 1(\bmod 2), \quad l \leq n .
\end{array}
$$


Theorem 1. In

$$
\begin{gathered}
v_{n l}(\theta, \varphi)=\sqrt{\frac{2(n-l) !}{\delta_{l}(n+l) !}} \cos (l \varphi) P_{n}^{(l)}(\cos \theta), \\
v_{n l}^{\prime}\left(\theta^{\prime}, \varphi^{\prime}\right)=\sqrt{\frac{2(n-l) !}{\delta_{l}(n+l) !}} \cos \left(l \varphi^{\prime}\right) P_{n}^{(l)}\left(\cos \theta^{\prime}\right), \quad l \leq n
\end{gathered}
$$

are spherical harmonics, then for the even spherical functions we can use the following formulas

$$
\begin{aligned}
& \sum_{l \equiv 1(\bmod 2)}^{n} v_{n l} v_{n l}^{\prime}=L_{1}\left(P_{n}(\cos \alpha)\right), \\
& \sum_{l \equiv 0(\bmod 2)}^{n} v_{n l} v_{n l}^{\prime}=L_{2}\left(P_{n}(\cos \alpha)\right),
\end{aligned}
$$

where

$$
\begin{aligned}
& L_{1} \equiv \sum_{m=0}^{n}\left|\sin \frac{\pi m}{2}\right| \prod_{k=0, k \neq m}^{n} \frac{-\frac{d^{2}}{d \varphi^{2}}-k^{2}}{m^{2}-k^{2}}, \\
& L_{2} \equiv \sum_{m=0}^{n}\left|\cos \frac{\pi m}{2}\right| \prod_{k=0, k \neq m}^{n} \frac{-\frac{d^{2}}{d \varphi^{2}}-k^{2}}{m^{2}-k^{2}} .
\end{aligned}
$$

Proof. In order to prove the theorem, we consider the following relations $[12,14]$

$$
\begin{aligned}
& L_{1}(\cos (l \varphi))=0, \quad l \equiv 0(\bmod 2), \\
& L_{1}(\cos (l \varphi))=\cos (l \varphi), \quad l \equiv 1(\bmod 2), \\
& L_{1}(\sin (l \varphi))=0, \quad l \equiv 0(\bmod 2) \text {, } \\
& L_{1}(\sin (l \varphi))=\sin (l \varphi), \quad l \equiv 1(\bmod 2), \\
& L_{2}(\cos (l \varphi))=0, \quad l \equiv 1(\bmod 2), \\
& L_{2}(\cos (l \varphi))=\cos (l \varphi), \quad l \equiv 0(\bmod 2), \\
& L_{2}(\sin (l \varphi))=0, \quad l \equiv 1(\bmod 2) \text {, } \\
& L_{2}(\sin (l \varphi))=\sin (l \varphi), \quad l \equiv 0(\bmod 2),
\end{aligned}
$$

which follow from the sequence of parities

$$
\begin{gathered}
L_{1}(\cos (l \varphi))=\sum_{m=0}^{n}\left|\sin \frac{\pi m}{2}\right| \prod_{k=0, k \neq m}^{n} \frac{l^{2}-k^{2}}{m^{2}-k^{2}} \cos (l \varphi)= \\
=\sum_{m \equiv 1(\bmod 2)}^{n} \prod_{k=0, k \neq m}^{n} \frac{l^{2}-k^{2}}{m^{2}-k^{2}} \cos (l \varphi)= \\
=\left\{\begin{array}{cc}
0, & l \equiv 0(\bmod 2), \\
\cos (l \varphi) \prod_{k=0}^{n} \frac{l^{2}-k^{2}}{l^{2}-k^{2}}, & l \equiv 1(\bmod 2)
\end{array}= \begin{cases}0, & l \equiv 0(\bmod 2), \\
\cos (l \varphi), & l \equiv 1(\bmod 2) .\end{cases} \right. \\
L_{1}(\sin (l \varphi))=\sum_{m=0}^{n}\left|\sin \frac{\pi m}{2}\right| \prod_{k=0, k \neq m}^{n} \frac{l^{2}-k^{2}}{m^{2}-k^{2}} \sin (l \varphi)=
\end{gathered}
$$




$$
\begin{aligned}
& =\sum_{m \equiv 1(\bmod 2)}^{n} \prod_{k=0, k \neq m}^{n} \frac{l^{2}-k^{2}}{m^{2}-k^{2}} \sin (l \varphi)= \\
& =\left\{\begin{array}{ll}
0, & l \equiv 0(\bmod 2), \\
\sin (l \varphi) \prod_{k=0}^{n} \frac{l^{2}-k^{2}}{l^{2}-k^{2}}, & l \equiv 1(\bmod 2)
\end{array}= \begin{cases}0, & l \equiv 0(\bmod 2), \\
\sin (l \varphi), & l \equiv 1(\bmod 2) .\end{cases} \right. \\
& L_{2}(\cos (l \varphi))=\sum_{m=0}^{n}\left|\cos \frac{\pi m}{2}\right| \prod_{k=0, k \neq m}^{n} \frac{l^{2}-k^{2}}{m^{2}-k^{2}} \cos (l \varphi)= \\
& =\sum_{m \equiv 0(\bmod 2)}^{n} \prod_{k=0, k \neq m}^{n} \frac{l^{2}-k^{2}}{m^{2}-k^{2}} \cos (l \varphi)= \\
& =\left\{\begin{array}{ll}
0, & l \equiv 1(\bmod 2), \\
\cos (l \varphi) \prod_{k=0}^{n} \frac{l^{2}-k^{2}}{l^{2}-k^{2}}, & l \equiv 0(\bmod 2)
\end{array}= \begin{cases}0, & l \equiv 1(\bmod 2), \\
\cos (l \varphi), & l \equiv 0(\bmod 2),\end{cases} \right. \\
& L_{2}(\sin l \varphi)=\sum_{m=0}^{n}\left|\sin \frac{\pi m}{2}\right| \prod_{k=0, k \neq m}^{n} \frac{l^{2}-k^{2}}{m^{2}-k^{2}} \sin (l \varphi)= \\
& =\sum_{m \equiv 0(\bmod 2)}^{n} \prod_{k=0, k \neq m}^{n} \frac{l^{2}-k^{2}}{m^{2}-k^{2}} \sin (l \varphi)= \\
& =\left\{\begin{array}{ll}
0, & l \equiv 1(\bmod 2), \\
\sin (l \varphi) \prod_{k=0}^{n} \frac{l^{2}-k^{2}}{l^{2}-k^{2}}, & l \equiv 0(\bmod 2)
\end{array}= \begin{cases}0, & l \equiv 1(\bmod 2), \\
\sin (l \varphi), & l \equiv 0(\bmod 2) .\end{cases} \right.
\end{aligned}
$$

Then

$$
\begin{aligned}
& \sum_{l=0}^{n} v_{n l} v_{n l}^{\prime}=2 \sum_{l=0}^{n} \frac{(n-l) !}{\delta_{l}(n+l) !} P_{n}^{(l)}(\cos \theta) P_{n}^{(l)}\left(\cos \theta^{\prime}\right) \cos (l \varphi) \cos \left(l \varphi^{\prime}\right)= \\
& =\sum_{l=0}^{n} \frac{2(n-l) !}{\delta_{l}(n+l) !} P_{n}^{(l)}(\cos \theta) P_{n}^{(l)}\left(\cos \theta^{\prime}\right)\left[\cos l\left(\varphi+\varphi^{\prime}\right)+\cos l\left(\varphi-\varphi^{\prime}\right)\right] .
\end{aligned}
$$

After replacing variable $\varphi^{\prime} \rightarrow 2 \pi-\varphi^{\prime}$, we get that [7]

$$
\cos l\left(\varphi+\varphi^{\prime}\right)=\cos l\left(\varphi+2 \pi-\varphi^{\prime}\right)=\cos l\left(\varphi-\varphi^{\prime}\right) \text {. }
$$

Therefore

$$
\sum_{l=0}^{n} v_{n l} v_{n l}^{\prime}=2 \sum_{l=0}^{n} \frac{2(n-l) !}{\delta_{l}(n+l) !} P_{n}^{(l)}(\cos \theta) P_{n}^{(l)}\left(\cos \theta^{\prime}\right) \cos l\left(\varphi-\varphi^{\prime}\right)
$$

After using the theorem [2], we get that

$$
\sum_{l=0}^{n} v_{n l} v_{n l}^{\prime}=P_{n}(\cos \alpha)
$$


it is obvious that

$$
\begin{aligned}
& L_{1}\left(\sum_{l=0}^{n} v_{n l} v_{n l}^{\prime}\right)=\sum_{l \equiv 1(\bmod 2)}^{n} v_{n l} v_{n l}^{\prime}, \\
& L_{2}\left(\sum_{l=0}^{n} v_{n l} v_{n l}^{\prime}\right)=\sum_{l \equiv 0(\bmod 2)}^{n} v_{n l} v_{n l}^{\prime} .
\end{aligned}
$$

Therefore $[6,8]$

$$
\begin{aligned}
& \sum_{l \equiv 1(\bmod 2)}^{n} v_{n l} v_{n l}^{\prime}=L_{1}\left(P_{n}(\cos \alpha)\right), \\
& \sum_{l \equiv 0(\bmod 2)}^{n} v_{n l} v_{n l}^{\prime}=L_{2}\left(P_{n}(\cos \alpha)\right) .
\end{aligned}
$$

\section{Analysis of the Perturbation Theory Corrections}

Let $T$ be Laplace - Bochner with potential on the projective plane $F$, acting on a Hilbert space $H$ of functions square-integrable with respect to Haar: $\sin \theta d \theta d \varphi(\theta, \varphi$ are spherical coordinates $), \lambda_{n}=n(n+1) \quad(n=\overline{0, \infty})$ - the eigenvalues of the operator $T$, $V n=2 n+1$ - the multiplicity of $\lambda_{n} ; V n, i \quad(i=\overline{0,2 n})$ - eigenfunctions $T$ forming an orthonormal system of spherical functions. Assume also that $l_{n}=\left\{\lambda \mid \lambda=\lambda_{n}+n+1+\right.$ $i \rho, \quad-\infty<\rho<\infty\}$ - vertical lines in the complex plane. We denote eigenvalues of $T+P$ by $\mu_{n, i}$, combined with the algebraic multiplicity such that

$$
\left|\mu_{n, i}-n(n+1)\right| \leq \text { const. }
$$

It is clear that

$$
\sum_{i=0}^{2 n} \mu_{n, i}=n(n+1)(2 n+1)+\sum_{i=0}^{2 n}\left(P v_{n i}, v_{n i}\right)+\alpha_{n}(p)+\beta_{n}(p)+O\left(\frac{1}{n^{2}}\right),
$$

where $\alpha_{n}(p)$ is the second amendment to the amount of perturbation theory $\sum_{i=0}^{2 n} \mu_{n, i}, \beta_{n}(p)$ - the third amendment of the perturbation theory. The first amendment is a constant [10]

$$
\sum_{i=0}^{2 n}\left(P v_{n i}, v_{n i}\right)=\frac{2 m+1}{4 \pi} \iint_{F} p(\theta, \varphi) \sin \theta d \varphi d \theta=\text { const. }
$$

The second amendment of the perturbation theory has the form

$$
\begin{gathered}
\alpha_{n}(p)=-\frac{1}{2 \pi i} S p\left\{\left[\int_{l_{n}}-\int_{l_{n}-1}\right] \lambda\left[(T-\lambda E)^{-1} P^{2}\right](T-\lambda E)^{-1} d \lambda\right\}=\sum_{k=1, k \neq n}^{\infty} \frac{\alpha_{k, n}}{\left|\lambda_{k}-\lambda_{n}\right|} \\
\alpha_{k, n}=\sum_{i=0}^{2 k} \sum_{j=0}^{2 n}\left(P v_{k i}, v_{n j}^{\prime}\right)\left(P v_{n j}, v_{k i}^{\prime}\right) .
\end{gathered}
$$


In addition theorem for even spherical harmonics [3]

$$
\begin{gathered}
\alpha_{k, n}=\sum_{i=0}^{2 k} \sum_{j=0}^{2 n}\left(P v_{k i}, v_{n j}^{\prime}\right)\left(P v_{n j}, v_{k i}^{\prime}\right)= \\
=\frac{(2 k+1)(2 n+1)}{4 \pi^{2}} \sum_{i=0}^{2 k} \sum_{j=0}^{2 n} \iint_{F} \iint_{F} p(\theta, \varphi) v_{k i}(\theta, \varphi) v_{n j}(\theta, \varphi) \sin (\theta) d \theta d \varphi \times \\
\times p\left(\theta^{\prime}, \varphi^{\prime}\right) v_{n j}^{\prime}\left(\theta^{\prime}, \varphi^{\prime}\right) v_{k i}^{\prime}\left(\theta^{\prime}, \varphi^{\prime}\right) \sin \left(\theta^{\prime}\right) d \theta^{\prime} d \varphi^{\prime}= \\
=\frac{(2 k+1)(2 n+1)}{4 \pi^{2}} \iint_{F} \iint_{F} p(\theta, \varphi) p\left(\theta^{\prime}, \varphi^{\prime}\right) \sin (\theta) \sin \left(\theta^{\prime}\right) \times \\
\times \sum_{i=0}^{2 k} v_{k i}(\theta, \varphi) v_{k i}^{\prime}\left(\theta^{\prime}, \varphi^{\prime}\right) \sum_{i=0}^{2 n} v_{n j}(\theta, \varphi) v_{n j}^{\prime}\left(\theta^{\prime}, \varphi^{\prime}\right) d \theta d \varphi d \theta^{\prime} d \varphi^{\prime}= \\
=\frac{(2 k+1)(2 n+1)}{4 \pi^{2}} \iint_{F} \iint_{F} p(\theta, \varphi) p\left(\theta^{\prime}, \varphi^{\prime}\right) \sin (\theta) \sin \left(\theta^{\prime}\right) \times \\
\times\left[L_{1}\left(P_{2 k}(\cos \alpha)\right)+L_{2}\left(P_{2 k}(\cos \alpha)\right)\right] \times \\
\times\left[L_{1}\left(P_{2 n}(\cos \alpha)\right)+L_{2}\left(P_{2 n}(\cos \alpha)\right)\right] d \theta d \varphi d \theta^{\prime} d \varphi^{\prime}= \\
=\frac{(2 k+1)(2 n+1)}{4 \pi^{2}} \iint_{F} \int_{F} p(\theta, \varphi) p\left(\theta^{\prime}, \varphi^{\prime}\right) \sin (\theta) \sin \left(\theta^{\prime}\right) \times \\
\times \sum_{i=1}^{2} L_{i}\left(P_{2 k}(\cos \alpha)\right) \sum_{i=1}^{2} L_{i}\left(P_{2 n}(\cos \alpha)\right) d \theta d \varphi d \theta^{\prime} d \varphi^{\prime}
\end{gathered}
$$

where

$$
\cos \alpha=\cos \theta \cos \theta^{\prime}+\sin \theta \sin \theta^{\prime} \cos \left(\varphi-\varphi^{\prime}\right) .
$$

Legendre polynomials $P_{2 k}, P_{2 n}$ normalized by the condition $P_{2 k}(1)=P_{2 n}(1)=1$. Define the function

$$
f(\alpha)=\iint_{F} p(\theta, \varphi) \sin \theta d \theta d \varphi\left[\iint_{T(\alpha)} p\left(\theta^{\prime}, \varphi^{\prime}\right) \sin \theta^{\prime} \frac{\phi\left(\theta, \theta^{\prime}, \alpha\right) d \theta^{\prime}}{\sin \alpha}\right],
$$

where $T(\alpha)$ is an intersection of the cone with apex at the center of the sphere central angle, $2 \alpha(0 \leqslant \alpha \leqslant \pi$ and an axis is defined by spherical coordinates $\theta, \varphi$, with a sphere in spherical coordinates $\theta^{\prime}, \varphi^{\prime}$;

$$
\begin{aligned}
\phi\left(\theta, \theta^{\prime}, \alpha\right) & = \pm \frac{\sin \alpha}{\sqrt{\left(-\cos \alpha+\cos \left(\theta-\theta^{\prime}\right)\right)\left(\cos \alpha-\cos \left(\theta+\theta^{\prime}\right)\right)}} \\
L_{j} & =L_{j}\left(\frac{d}{d \theta}\right), L_{j}^{\prime}=L_{j}\left(\frac{d}{d \theta^{\prime}}\right),(j=1,2)
\end{aligned}
$$


By hypothesis, the function $f$ is Lipschitz. Therefore the function is also Lipschitz:

$$
\begin{gathered}
|f(\alpha)-f(\beta)|=\iint_{F} p(\theta, \varphi) L_{j} \sin \theta d \theta d \varphi \times \\
\times\left[\iint_{T(\alpha)} p\left(\theta^{\prime}, \varphi^{\prime}\right) L_{j}^{\prime} \sin \theta^{\prime} \phi\left(\theta, \theta^{\prime}, \alpha\right) d \theta^{\prime}-\iint_{T(\alpha)} p\left(\theta^{\prime}, \varphi^{\prime}\right) L_{j}^{\prime} \sin \theta^{\prime} \phi\left(\theta, \theta^{\prime}, \alpha\right) d \theta^{\prime}\right] \leq \\
\leq \text { const }|\alpha-\beta| .
\end{gathered}
$$

Hence the function $f$ is absolutely continuous. Therefore at each point $[0, \pi]$ it has a finite derivative $f^{\prime}(\alpha)$, which is an integrable function.

Based on the foregoing, the second correction of perturbation theory has the form $(\varepsilon>0$ will be chosen later)

$$
\begin{gathered}
\alpha_{n}(p)=-\frac{(2 n+1)}{4 \pi^{2}} \sum_{k=1, k \neq n}^{\infty} \frac{(2 k+1)}{\left|\lambda_{k}-\lambda_{n}\right|} \int_{0}^{\pi} f(\alpha) P_{2 k}(\cos \alpha) P_{2 n}(\cos \alpha) \sin \alpha d \alpha= \\
=-\frac{(2 n+1)}{4 \pi^{2}} \sum_{k=1, k \neq n}^{\infty} \frac{(2 k+1)}{\left|\lambda_{k}-\lambda_{n}\right|}\left\{\left[\int_{0}^{\varepsilon}+\int_{\pi-\varepsilon}^{\pi}\right] f(\alpha) P_{2 k}(\cos \alpha) P_{2 n}(\cos \alpha) \sin \alpha d \alpha+\right. \\
\left.+\int_{\varepsilon}^{\pi-\varepsilon} f(\alpha) P_{2 k}(\cos \alpha) P_{2 n}(\cos \alpha) \sin \alpha d \alpha\right\} .
\end{gathered}
$$

For Legendre polynomials known [1] asymptotic expansion Stieltjes uniform remainder estimate

$$
\begin{gathered}
P_{k}(\cos \alpha)=\frac{\cos \{(2 k+1 / 2) \alpha-\pi / 4\}}{(\sin \alpha)^{1 / 2}}\left[\frac{(2 / \pi)^{1 / 2}}{2 k^{1 / 2}}+\frac{O(1)}{2 k^{3 / 2}}\right]+ \\
+\frac{\sin \{(2 k+3 / 2) \alpha-\pi / 4\}}{(\sin \alpha)^{3 / 2}} \frac{O(1)}{2 k^{3 / 2}}+\frac{O(1)}{(\sin \alpha)^{5 / 2} 2 k^{5 / 2}} .
\end{gathered}
$$

Using this asymptotic expansion, we transform $\alpha_{n}(p)$ as follows:

$$
\begin{aligned}
\alpha_{n}(p)=-\frac{(2 n+1)}{4 \pi^{2}} \sum_{k=1, k \neq n}^{\infty} \frac{(2 k+1)}{\left|\lambda_{k}-\lambda_{n}\right|}\left\{\left[\int_{0}^{\varepsilon}+\int_{\pi-\varepsilon}^{\pi}\right] f(\alpha) \sin \alpha P_{2 k}(\cos \alpha) P_{2 n}(\cos \alpha) d \alpha+\right. \\
+\int_{\varepsilon}^{\pi-\varepsilon} f(\alpha) \cos \{(2 k+1 / 2) \alpha-\pi / 4\} \cos \{(2 n+1 / 2) \alpha-\pi / 4\} \times \\
\quad \times\left\{\frac{2 / \pi}{k^{1 / 2} n^{1 / 2}}+\frac{O(1)}{k^{3 / 2} n^{1 / 2}}+\frac{O(1)}{k^{1 / 2} n^{3 / 2}}+\frac{O(1)}{k^{3 / 2} n^{3 / 2}}\right\} d \alpha+ \\
+\int_{\varepsilon}^{\pi-\varepsilon} \frac{f(\alpha)}{\sin \alpha} \cos \{(2 k+1 / 2) \alpha-\pi / 4\} \sin \{(2 n+3 / 2) \alpha-\pi / 4\} \times
\end{aligned}
$$




$$
\begin{gathered}
\times\left\{\frac{O(1)}{k^{1 / 2} n^{3 / 2}}+\frac{O(1)}{k^{3 / 2} n^{3 / 2}}\right\} d \alpha+ \\
+\int_{\varepsilon}^{\pi-\varepsilon} \frac{f(\alpha)}{\sin ^{2} \alpha} \cos \{(2 k+1 / 2) \alpha-\pi / 4\} \frac{O(1)}{k^{1 / 2} n^{5 / 2}} d \alpha+ \\
+\int_{\varepsilon}^{\pi-\varepsilon} \frac{f(\alpha)}{\sin \alpha} \sin \{(2 k+3 / 2) \alpha-\pi / 4\} \times \\
+\int_{\varepsilon}^{\pi-\varepsilon} \frac{f(\alpha)}{\sin ^{2} \alpha} \sin \{(2 k+3 / 2) \alpha-\pi / 4\} \sin \{(2 n+3 / 2) \alpha-\pi / 4\} \frac{O(1)}{k^{3 / 2} n^{3 / 2}} d \alpha+ \\
+\int_{\varepsilon}^{\pi-\varepsilon} \frac{f(\alpha)}{\sin ^{3} \alpha} \sin \{(2 k+3 / 2) \alpha-\pi / 4\} \frac{O(1)}{k^{3 / 2} n^{5 / 2}} d \alpha+ \\
+\int_{\varepsilon}^{\pi-\varepsilon} \frac{f(\alpha)}{\sin ^{2} \alpha} \cos \{(2 k+1 / 2) \alpha-\pi / 4\} \frac{O(1)}{k^{1 / 2} n^{5 / 2}} d \alpha+ \\
\left.+\int_{\varepsilon}^{\pi-\varepsilon} \frac{f(\alpha)}{\sin ^{3} \alpha} \sin \{(2 n+3 / 2) \alpha-\pi / 4\} \frac{O(1)}{k^{5 / 2} n^{3 / 2}} d \alpha+\int_{\varepsilon}^{\pi-\varepsilon} \frac{f(\alpha)}{\sin ^{4} \alpha} \frac{O(1)}{k^{5 / 2} n^{5 / 2}} d \alpha\right\}
\end{gathered} .
$$

Finally,

$$
\begin{gathered}
\alpha_{n}(p)=O\left(\varepsilon^{2} \ln n\right)+O\left(\frac{\varepsilon \ln n}{n}\right)+O\left(\frac{\varepsilon}{n^{3 / 2}}\right)+O\left(\frac{\varepsilon}{n}\right)+ \\
+O\left(\frac{\varepsilon \ln n}{n^{2}}\right)+O\left(\frac{\varepsilon \ln n}{n^{2}}\right)+O\left(\frac{\varepsilon}{n^{2}}\right)+O\left(\frac{\varepsilon \ln n}{n^{2}}\right)- \\
-\frac{1}{4 \pi^{2} n} \int_{\varepsilon}^{\pi-\varepsilon} f(\alpha) \operatorname{ctg} \alpha(\pi-\alpha) d \alpha+O\left(\frac{\varepsilon \ln n}{n^{2}}\right)+O\left(\frac{\ln n}{n^{3 / 2}}\right)+O\left(\frac{\ln \varepsilon \ln n}{n^{3 / 2}}\right)+ \\
+O\left(\frac{\ln n}{n^{3} \varepsilon}\right)+O\left(\frac{\ln \varepsilon}{n^{3 / 2}}\right)+O\left(\frac{1}{\varepsilon n^{3 / 2}}\right)+O\left(\frac{1}{n^{3} \varepsilon^{3}}\right) .
\end{gathered}
$$

Choose $\varepsilon=\frac{1}{n^{3 / 4}}$ and find

$$
\alpha_{n}(p)=\frac{1}{4 \pi^{2} n} \int_{\varepsilon}^{\pi-\varepsilon} f(\alpha) \operatorname{ctg} \alpha(\pi-\alpha) d \alpha+O\left(\frac{\ln n}{n^{3 / 2}}\right) .
$$

The third amendment of the perturbation theory

$$
\beta_{n}(p)=\frac{1}{6 \pi i} S p\left[\int_{l_{n}}-\int_{l_{n-1}}\right]\left[P(T-\lambda E)^{-1}\right]^{3} d \lambda=
$$




$$
\begin{gathered}
=\frac{1}{6 \pi i}\left[\int_{l_{n}}-\int_{l_{n-1}}\right] \sum \frac{(2 n+1)(2 k+1)(2 l+1)}{\left(\lambda_{n}-\lambda\right)\left(\lambda_{k}-\lambda\right)\left(\lambda_{l}-\lambda\right)} d \lambda \times \\
\times\left(\frac{1}{4 \pi}\right)^{3} \iint_{F} \iint_{F} \iint_{F} p(\theta, \varphi) p\left(\theta^{\prime}, \varphi^{\prime}\right) p\left(\theta^{\prime \prime}, \varphi^{\prime \prime}\right) \times \\
\quad \times P_{n}(\cos \alpha) P_{n}(\cos \beta) P_{n}(\cos \gamma) \times \\
\times \sin \theta \sin \theta^{\prime} \sin \theta^{\prime \prime} d \varphi d \varphi^{\prime} d \varphi^{\prime \prime} d \theta d \theta^{\prime} d \theta^{\prime \prime}, \\
\cos \alpha=\cos \theta \cos \theta^{\prime}+\sin \theta \sin \theta^{\prime} \cos \left(\varphi--\varphi^{\prime}\right), \\
\cos \beta=\cos \theta^{\prime} \cos \theta^{\prime \prime}+\sin \theta^{\prime} \sin \theta^{\prime \prime} \cos \left(\varphi^{\prime}--\varphi^{\prime \prime}\right), \\
\cos \gamma=\cos \theta \cos \theta^{\prime \prime}+\sin \theta \sin \theta^{\prime \prime} \cos \left(\varphi--\varphi^{\prime \prime}\right) .
\end{gathered}
$$

Finally,

$$
\beta_{n}(p)=0
$$

Main theorem.

Theorem 2. If $p$ - potential Lipschitz two variables, then the eigenvalues $T+P$ the equality

$$
\sum_{i=0}^{2 n} \mu_{n, i}-n(n+1)(2 n+1)=O\left(\frac{\ln n}{n^{1 / 2}}\right) .
$$

Theorem 3. The first regularized trace for the Laplace - Bochner complex potential Lipschitz has the form

$$
\mu_{0,0}+\lim _{m \rightarrow \infty}\left(\sum_{n=1}^{m}\left\{\sum_{i=0}^{2 n} \mu_{n, i}-n(n+1)(2 n+1)\right\}\right)=\text { const. }
$$

And according to $k$ number of absolutely convergent.

Thus, we calculated the regularized trace for the Laplace - Bochner of a non-smooth potential, namely, the potential is Lipschitz.

\section{References}

1. Dubrovsky V.V., Torshina O.A. [Problem Solving Eigenvalue Problems for Differential Operators with a Complex Spectral Parameter]. Elektromagnitnye volny i elektronnye systemy [Electromagnetic Waves and Electronic Systems], 2002, vol. 7, no. 9, pp. 4-10. (in Russian)

2. Dubrovsky V.V., Torshina O.A. [The Formula for the First Regularized Trace of the Differential Laplace - Beltrami Operator]. Differential Equations and Their Applications, 2002, no. 1, pp. 9-19. (in Russian)

3. Torshina O.A. [The Algorithm for Calculation of the Regularized Trace for the Laplace - Beltrami Operator with Potential on the Projective Plane]. Vestnik MaGU. Matematika, 2003, vol. 4, pp. 183-215. (in Russian) 
4. Torshina O.A. [The Traces of Discrete Operators with Partial Derivatives]. Almanac of Modern Science and Education, 2012, no. 4 (59), pp. 220-222. (in Russian)

5. Torshina O.A. [The Formula for the First Regularized Trace of the Laplace - Bochner Operator with Potential on the Projective Plane]. Voronezh Winter Mathematical School, 2004, pp. 104-105. (in Russian)

6. Torshina O.A. [The Formula for the First Regularized Trace of the Laplace Bochner Operator with Potential on the Non-Smooth Projective Plane]. Vestnik Samarskogo gosudarstvennogo tekhnicheskogo universiteta. Matematika, 2006, pp. 240. (in Russian)

7. Torshina O.A. [The Formula of the Regularized Trace of a Differential Operator with Complex Spectral Parameter]. General Management Problems and Their Applications, 2003, pp. 67-468. (in Russian)

8. Torshina O.A. [A Numerical Method for Calculating Corrections of Perturbation Theory]. Almanac of Modern Science and Education, 2013, no. 12, pp. 168-170. (in Russian)

9. Dubrovskii V.V., Kadchenko S.I., Kravchenko V.F., Sadovnichii V.A. A New Method for Approximate Evaluation of the First Eigenvalues in the Orr - Sommerfelg Eigenvalue Problem. Doklady Mathematics, 2001, vol. 63, no. 3, pp. 355-358.

10. Dubrovskii V.V., Kadchenko S.I., Kravchenko V.F., Sadovnichii V.A. A New Method for Approximate Evaluation of the First Eigenvalues in the Spectral Problem of Hydrodynamic Stability of Poiseuille Flow in a Circular Pipe. Doklady Mathematics, 2001, vol. 64, no. 2, pp. 165-168.

11. Dubrovskii V.V., Kadchenko S.I., Kravchenko V.F., Sadovnichii V.A. A New Method for the Evaluation of the First Eigenvalues in the Spectral Problem of Hydrodynamic Stability of Viscous Fluid Flow between Two Rotating Cylinders. Doklady Mathematics, 2001, vol. 64, no. 3, pp. 425-429.

12. Dubrovskii V.V., Kadchenko S.I., Kravchenko V.F., Sadovnichii V.A. Computation of the First Eigenvalues of a Discrete Operator. Elektromagnitnye volny $i$ elektronnye systemy [Electromagnetic Waves and Electronic Systems], 1998, vol. 3, no. 2, pp. 4.

13. Sadovnichii V.A., Dubrovskii V.V., Kadchenko S.I., Kravchenko V.F. Evaluation of Eigenvalues of the Problem of Hydrodynamic Stability of Viscous Liquid Flow Between Two Rotating Cylinders at Small Reynolds Numbers. Doklady Mathematics, 1998, vol. 58 , no. 3, pp. $483-486$.

14. Sadovnichii V.A., Dubrovskii V.V., Kadchenko S.I., Kravchenko V.F. Computation of the First Eigenvalues of the Hydrodynamic Stability Problem for a Viscous Fluid Flow Between Two Rotating Cylinders. Differential Equations, 2000, vol. 36, p. 819.

Olga A. Torshina, candidate of physical and mathematical sciences, associate professor, department of Applied Mathematics and Computer Science, Nosov Magnitogorsk State Technical University, Magnitogorsk, Russian Federation, olganica@mail.ru.

Received October 30, 2015 


\title{
ДИФФЕРЕНЦИАЛЬНЫЕ ОПЕРАТОРЫ НА ПРОЕКТИВНОЙ ПЛОСКОСТИ
}

\author{
O.A. Торшина
}

\begin{abstract}
Ранее была получена формула сложения для четных сферических гармоник, которая послужила основой для вывода формулы первого регуляризованного следа оператора Лапласа с потенциалом на проективной плоскости. Благодаря ей удалось обойти нахождение асимптотических формул для присоединенных полиномов Лежандра по трем параметрам, что являлось неразрешимой задачей в течение длительного времени. Полученные результаты стали основой для вычисления поправок теории возмущений с последующим выходом на формулы регуляризованных следов эллиптических дифференциальных операторов. В работе рассматривается задача суммирования класса расходящихся рядов. Предлагается метод вычисления поправок теории возмущений для дифференциального оператора с потенциалом на действительной проективной плоскости. Метод применим, в частности, для суммирования рядов с факториальным ростом членов.
\end{abstract}

Ключевые слова: оператор Лапласа - Бохнера; проективная плоскость; гилъбертово пространство; полином Лежандра; условие Липшича.

\section{Литература}

1. Дубровский, В.В. Проблема решения задач на собственные значения для дифференциальных операторов со сложным вхождением спектрального параметра / В.В. Дубровский, О.А. Торшина // Электромагнитные волны и электронные системы - 2002. - Т. 7, № 9. - С. 4-10.

2. Дубровский, В.В. Формула первого регуляризованного следа для дифференциального оператора Лапласа - Бельтрами / В.В. Дубровский, О.А. Торшина // Дифференциальные уравнения и их приложения. - 2002. - № 1. - С. 9-19.

3. Торшина, О.А. Алгоритм вычисления регуляризованного следа оператора Лапласа - Бельтрами с потенциалом на проективной плоскости / О.А. Торшина // Вестник МаГУ. Математика. - 2003. - Вып. 4. - С. 183-215.

4. Торшина, О.А. Следы дискретных операторов с частными производными О.А. Торшина // Альманах современной науки и образования. - 2012. - № 4 (59). C. $220-222$.

5. Торшина, О.А. Формула первого регуляризованного следа Лапласа - Бохнера с потенциалом на проективной плоскости / О.А. Торшина // Воронежская зимняя математическая школа. - 2004. - С. 104-105.

6. Торшина, О.А. Формула первого регуляризованного следа оператора Лапласа Бельтрами с негладким потенциалом на проективной плоскости / О.А. Торшина // Вестник Самарского государственного технического университета. Математика. - 2006. - С. 32-40.

7. Торшина, О.А. Формула регуляризованного следа дифференциального оператора со сложным вхождением спектрального параметра / О.А. Торшина // Общие проблемы управления и их приложения. - 2003. - С. 467-468. 
8. Торшина, О.А. Численный метод вычисления поправок теории возмущений О.А. Торшина // Альманах современной науки и образования. - 2013. - № 12. C. $168-170$.

9. Dubrovskii, V.V. A New Metod for Approximate Evaluation of the First Eigenvalues in the Orr - Sommerfeld Eigenvalue Problem / V.V. Dubrovskii, S.I. Kadchenko, V.F. Kravchenko, V.A. Sadovnichii // Doklady Mathematics. - 2001. - V. 63, № 3.P. 355-358.

10. Dubrovskii, V.V. A New Method for Approximate Evaluation of the First Eigenvalues in the Spectral Problem of Hydrodynamic Stability of Poiseuille Flow in a Circular Pipe / V.V. Dubrovskii, S.I. Kadchenko, V.F. Kravchenko, V.A. Sadovnichii // Doklady Mathematics. - 2001. - V. 64, № 2. - P. 165-168.

11. Dubrovskii, V.V. A New Method for the Evaluation of the First Eigenvalues in the Spectral Problem of Hydrodynamic Stability of Viscous Fluid Flow Between Two Rotating Cylinders / V.V. Dubrovskii, S.I. Kadchenko, V.F. Kravchenko, V.A. Sadovnichii // Doklady Mathematics. - 2001. - V. 64, № 3. - P. 425-429.

12. Dubrovskii, V.V. Computation of the First Eigenvalues of a Discrete Operator / V.V. Dubrovskii, S.I. Kadchenko, V.F. Kravchenko, V.A. Sadovnichii // Электромагнитные волны и электронные системы. - 1998. - Т. 3, № 2. - С. 4.

13. Sadovnichii, V.A. Evaluation of Eigenvalues of the Problem of Hydrodynamic Stability of Viscous Liquid Flow Between Two Rotating Cylinders at Small Reynolds Numbers / V.A. Sadovnichii, V.V. Dubrovskii, S.I. Kadchenko, V.F. Kravchenko // Doklady Mathematics. - 1998. - V. 58, № 3. - P. 483-486.

14. Sadovnichii, V.A. Computation of the First Eigenvalues of the Hydrodynamic Stability Problem for a Viscous Fluid Flow Between Two Rotating Cylinders // V.A. Sadovnichii, V.V. Dubrovskii, S.I. Kadchenko, V.F. Kravchenko // Differential Equations. - 2000. - V. 36. - P. 819.

Ториина Ольга Анатольевна, кандидат физико-математических наук, дочент, кафедра прикладной математики и информатики, Магнитогорский государственный технический университет им. Г.И. Носова (г. Магнитогорск, Российская Федерация),olganica@mail.ru

Поступила в редакиию 30 октября 2015. 